\title{
LOCAL RECOGNITION OF TITS GEOMETRIES OF CLASSICAL TYPE
}

\begin{abstract}
A method, based on Tits' work and involving an idea of M. Ronan, is developed in order to recognize certain geometries which are locally buildings of classical type as quotients of buildings. Two applications are treated in detail showing that every finite nearly classical near polygon must be a dual polar space and that in the finite case of Cooperstein's theorem characterizing geometries of Lie type $D_{n}$, the hypotheses can be weakened considerably.
\end{abstract}

\section{INTRODUCTION}

An interesting topic in the theory of incidence systems (i.e. geometries of rank 2) associated with buildings of spherical type is the local recognition problem. This is the question whether such an incidence system is fully determined by the structure induced on the set of points collinear with a given point. The only examples of studies strictly of this kind that we know of are Buekenhout [5], Buekenhout and Hubaut [7], Hall and Shult [13], Johnson and Shult [14], and Kantor [15], where certain locally polar spaces are characterized. In general, it seems that the problem is a very hard one. On the other hand, much has been achieved under mild extra conditions involving the structure on the set of points at distance at most 2 (measured in the collinearity graph) to a given point. Recently, in Cohen [10] and Cohen and Cooperstein [11], geometries of type $A_{n}, D_{n}, E_{6}, E_{7}, E_{8}$, and $F_{4}$ have been characterized in such a way. The classical result of Buekenhout and Shult [8] characterizing polar spaces (on which the above-mentioned local characterization of Johnson and Shult [14] is based) is both local and global in nature since it concerns an axiom on incidence systems that forces all distances in the collinearity graph to be at most 2 .

Two more beautiful characterizations of incidence systems associated with buildings are the theorems by Cameron [9] and Cooperstein [12] on dual polar spaces (type $C_{n}$ ) and halved dual polar spaces (type $D_{n}$ ), respectively. Both, however, use conditions involving points at arbitrarily large mutual distance. The general (and notably Shult's) feeling was that the hypotheses of both theorems might be weakened to conditions involving only points at mutual distance at most $m$ for some suitable natural number $m$. In [18] Shult describes a way to get rid of at least one of the global assumptions in Cameron's theorem. The main purpose of this article is to establish a version of the theorems of Cameron and of Cooperstein which only uses the part of their 
hypotheses involving points at mutual distance at most 3 and 2, respectively (see the Remarks after Corollary 2 and Corollary 3 below).

MAIN THEOREM. Let $\Gamma$ be an incidence system all of whose lines are thick and let $n$ be a natural number, $n \geqslant 4$.

(i) Suppose that $\Gamma$ is a nearly classical 3-weak near polygon (cf. Section 6). Suppose, moreover, that $\Gamma$ has finite local rank $n-1$ at some point. Then $\Gamma$ is isomorphic to the quotient $\Delta / A$ of a dual polar space of rank $n$ by a group $A$ of automorphisms of $\Delta$ such that for each $a \in A-\{1\}$ the distance between a point and its image under $a$ is at least 8 . In particular, any finite nearly classical near polygon is a dual polar space.

(ii) Suppose that $\Gamma$ is a parapolar space of singular rank $n-1 \geqslant 4$ satisfying $(P 3)_{3}$ and (P4) (cf. Section 6). Then $\Gamma$ is isomorphic to the quotient $\Delta / A$ of a halved dual polar space $\Delta$ by a group $A$ of automorphisms of $\Delta$ such that for each $a \in A-\{1\}$ the distance between a point and its image under $a$ is at least 5 . In particular, if $\Gamma$ is finite, then it is a halved dual polar space.

The proof of the main theorem uses neither Cameron [9] nor Cooperstein [12], but depends on Tits [20] and a sharpened version of Theorem 5 in Ronan [16] concerning reconstructions of truncated chamber systems (cf. Theorem 2). The finite case leads to a strengthening of their results, the infinite case leads to a slightly more general outcome to the extent that certain quotients of (halved) dual polar spaces occur.

The proofs are structured in such a way that analogous results for other geometries related to buildings are easily derived. For an example, see Theorem 4.

\section{Geometries}

Throughout this paper, $I$ stands for a finite index set of cardinality $n$. We recall some definitions from Tits [20] and Buekenhout [6].

A geometry over $I$ is a system $\Gamma=(V, *, t)$, consisting of a set $V$ of objects, a symmetric relation $*$ on $V$, called incidence, and a map $t: V \rightarrow I$, the type map, such that for any two elements $x, y$ of $V$ with $t(x)=t(y)$ the relation $x * y$ holds if and only if $x=y$. Usually, $I$ is called the type set of $\Gamma$ and $n$ is the rank of $\Gamma$. The tuple $(V, *)$ is called the (incidence) graph of $\Gamma$. (Notice that in this graph each vertex is in a unique loop.) Morphisms of geometries over $I$ are type and incidence preserving maps. Let $J$ be a subset of $I$. A flag of $\Gamma$ (of type $J$ ) is a set $F$ of pairwise incident objects of $\Gamma$ (such that $t(F)=J$ ). The cotype of a flag of type $J$ is $I-J$. The rank (corank) of a flag $F$ is the cardinality of $t(F)$ (respectively of $I-t(F))$. Let $F$ be a flag of type $J$, and let $W$ be the set of all elements of $V-F$ 
incident to $F$ (i.e. to each element of $F$ ). Then the $\operatorname{system}\left(W, \cap(W \times W),\left.t\right|_{W}\right)$, considered as a geometry over $I-J$, is called the residue of $F$ in $\Gamma$, and is denoted by $\Gamma_{F}$ or, if $\Gamma$ is clear from the context, by $\operatorname{Res}(F)$. Also, if $x$ is an object, we shall write $\Gamma_{x}$ rather than $\Gamma_{\{x\}}$. Thus, a residue of type $K$, for $K \subseteq I$, is the residue of a flag of type $I-K$. A geometry is called connected if its incidence graph is connected. It is called residually connected if, for each flag $F$ of corank at least $1, \operatorname{Res}(F)$ is nonempty and if, for each flag of corank at least $2, \operatorname{Res}(F)$ is connected.

For $J \subseteq 1$, set $K=I-J$ and $U=t^{-1}(K)$. The system,$\Gamma=(U, * \cap(U \times U)$, $\left.t\right|_{U}$ ) considered as a geometry over $K$, is called the $J$-truncation of $\Gamma$, or the truncation of $\Gamma$ of type $K$. Instead of ${ }_{J} \Gamma$, we shall also write ${ }^{\mathrm{K}} \Gamma$. Observe that each truncation of a residually connected geometry is itself residually connected (cf. Tits [20, Lemma 5]). A diagram over $I$ is a map $D$ assigning to each subset $H$ of $I$ of size 2 a class of rank 2 geometries over $H$. We say that $\Gamma$ is of type $D$ if it is residually connected and if for each subset $H$ of $I$ of size 2 each residue of type $H$ belongs to $D(H)$. Again, let $J$ be a subset of $I$. A geometry is said to be of $J$-truncated type $D$ if it is a residually connected geometry over $I-J$ such that for each subset $H$ of $I-J$ of size 2 every residue of type $H$ is the $J$-truncation of a geometry of type $\left.D\right|_{(J \cup X)}$. Here, the latter symbol denotes the restriction of $D$ to the collection of subsets of size 2 contained in $J \cup H$. Thus, if $\Gamma$ is a geometry of $J$-truncated type $D$, then for each flag $F$ of $\Gamma$ of cotype $H$ with $H \subseteq I-J$ of size 2 , there exists a geometry $\Sigma(F)$ of type $\left.D\right|_{(J \cup H)}$ such that $\Gamma_{F}=J_{J}(F)$. We shall make frequent use of the simple observation that, for $H \subseteq I-J$ a residue of type $H$ of a geometry of $J$-truncated type $D$ is a geometry of $J$-truncated type $\left.D\right|_{(J \cup H)}$.

A Coxter matrix over $I$ is a map $M: I \times I \rightarrow \mathbb{N}$ such that for $i, j \in I$ the relations $M(i, i)=1$ and $M(i, j)=M(j, i) \geqslant 2$ if $i \neq j$ hold. A Coxeter matrix $M$ over $I$ determines canonically a diagram $D=D_{M}$, the Coxeter diagram associated with $M$, where $D_{M}(\{i, j\})$ for any two distinct $i, j$ in $I$ is the class of all generalized $M(i, j)$-gons. (For $m \in \mathbb{N}$ and $m \geqslant 2$ a geometry of rank 2 is called a generalized $m-g o n$ if its incidence graph has diameter $m$ and girth $2 m$ and if every vertex of the graph belongs to at least two edges.)

Often we shall say that a geometry is of ( $J$-truncated) type $M$ when in fact $D_{M}$ is meant. Instead of giving the Coxeter matrix (or diagram) as a matrix, one usually draws a picture with the following conventions: draw a node (small circle) for each element $i$ of $i$; this node may be labelled $i$ for purposes of reference. For each (unordered) pair $i, j$ of distinct elements of $I$, join the nodes corresponding to $i$ and $j$ with an $(m-2)$-fold edge when $M(i, j)=m$.

We shall give names to some well-known Coxeter diagrams 


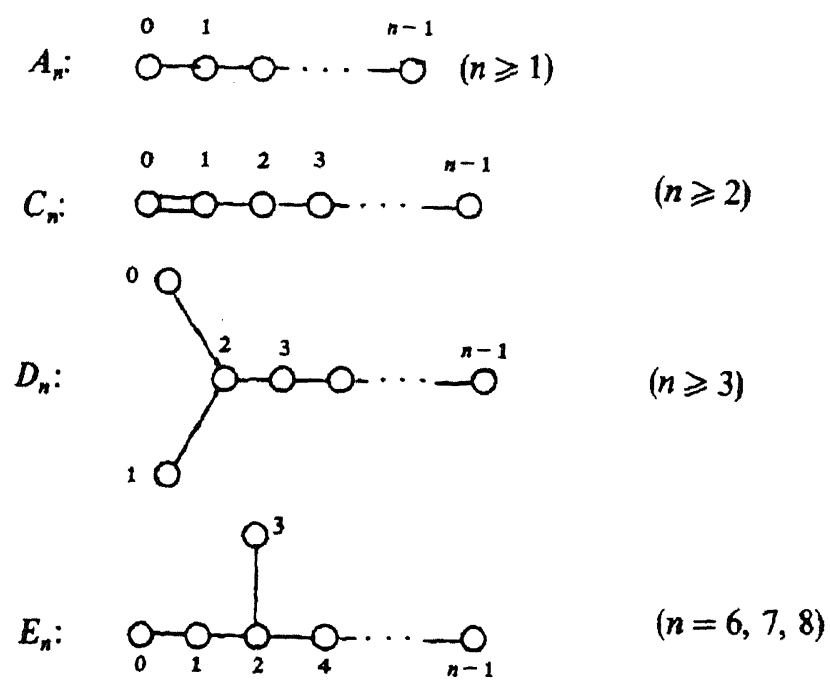

In such pictures, we shall single out a subset $J$ of $I$ for which we want to study $J$-truncated geometries of type $D_{M}$ by replacing the small circles of the nodes corresponding to members of $J$ by small squares. In this vein, a geometry of $\{3, \ldots, n-1\}$-truncated type $A_{n}$ will also be referred to as a geometry of type

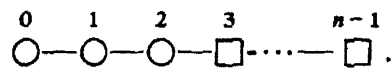

Thus, by definition, a geometry of type $\stackrel{0}{O}-\stackrel{1}{O}-\stackrel{2}{\square}^{-} \cdots \cdots-\square^{n-1}$ is the truncation of type $\{0,1\}$ of a geometry of type $A_{n}$.

Here is an easy example of the kind of result that we are after.

THEOREM 1. Let $I=\{0,1, \ldots, n-1\}$ and $J=\{3, \ldots, n-1\}$. Every geometry $\Gamma$ of J-truncated type $A_{n}$ is the J-truncation of a geometry $\Sigma$ of type $A_{n}$. Moreover, the geometry $\Sigma$ is unique in the sense that if $\Sigma^{1}$ is another such geometry, there is a unique isomorphism $\phi: \Sigma \rightarrow \Sigma^{1}$ of geometries which induces the identity on,$\Sigma=\Gamma={ }_{3} \Sigma^{1}$.

Proof. This is a straightforward consequence of Veblen and Young. Cf. Tits [20].

For the notion of building of type $M$, the reader is referred to Tits [20]. Here, we shall view such buildings as special kinds of geometries of type $M$. The following result is the analogue of Theorem 1 for (halved) dual polar spaces. It is a strengthened version of a theorem of Ronan [16] in that certain properties of the group $A$ are explicitly described. 
THEOREM 2. Let $I=\{0, \ldots, n-1\}$ and $J=\{4, \ldots, n-1\}$, where $n \geqslant 4$. Suppose $M=C_{n}$ or $D_{n}$. A geometry of J-truncated type $M$ (see the diagram below) such that each residue of type $C_{3}$ (i.e if $M=C_{n}$ each residue of type $\{0,1,2\}$ ) is covered by a building, is the J-truncation of the quotient geometry $\Delta / A$, where $\Delta$ is a building of type $M$ and $A$ is a group of automorphisms of $\Delta$ acting freely on the set of all flags of corank 2 and satisfying

(Q1) ${ }_{J} \quad$ The canonical projection $\pi: \Delta \rightarrow \Delta / A$ induces an isomorphism of the quotient $\Delta_{F} / A_{F}$ of the residue of $F$ by the stabilizer $A_{F}$ of $F$ in $A$ onto the residue $(\Delta / A)_{x(F)}$ for all flags $F$ of $\Delta$ with type not properly contained in $J$.

COROLLARY 1. Let $I$, $J$ be as above. Any thick finite geometry of $J$-truncated type $M=C_{n}$ or $D_{n}$ all of whose residues of type $C_{3}$ are covered by buildings, is the $J$-truncation of $a$ building of type $M$.

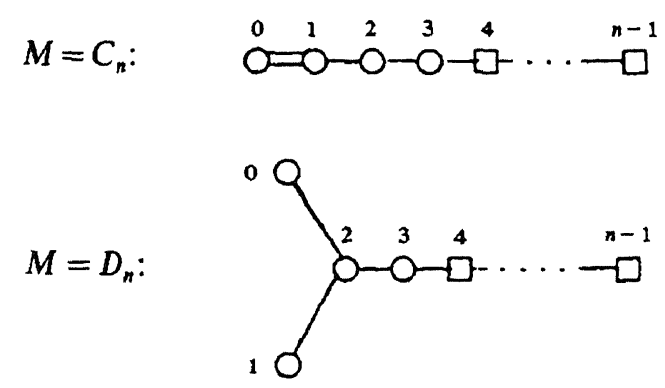

For convenience to the reader, the proof of Theorem 2 is given with references only to Tits [20], though part of it could have been taken from Ronan [16]. Our proof, though more geometric in nature than the one in [16], is based on the same idea of involving Tits' strong results on coverings of chamber systems.

REMARK. In Cohen and Cooperstein [11], examples have been given of quotient geometries of type $D_{n}$ (over $\mathbb{R}$ ) which also serve as examples of geometries of $J$-truncated type $D_{n}$ which are not $J$-truncations of buildings. (Here $J$ is as in the theorem.) Moreover, Ronan [16] exhibits a finite geometry of $\{3\}$-truncated type $C_{4}$ associated with the Mathieu group $M_{24}$ which is not the $\{3\}$-truncation of a building of type $C_{4}$. This shows that Theorem 2 is in a certain sense best possible.

\section{CHAMBER SYSTEMS}

Again, $I$ is an arbitrary index set of cardinality $n<\infty$. We first recall the notion mentioned in the heading of this section, which is introduced by Tits in [20]. 
A chamber system over $I$ consists of a set $C$, whose elements are called chambers, and a system $P=\left(P_{i}\right)_{i \in I}$ of partitions of $C$ indexed by $I$. For any subset $J$ of $I$, the $J$-graph of a chamber system $(C, P)$ over $I$ has vertex set $C$ and $c, d \in C$ are joined by an edge labelled $i$, i.e. are $i$-adjacent, if $i \in J$ and $c, d$ belong to the same element of $P_{i}$. In particular, the graph of $(C, P)$ is its $I$-graph. The chamber system is called connected whenever its graph is connected. A $J_{-}$ residue is an $(I-J)$-component of $C$, i.e. a connected component of its $(I-J)$ graph.

Notice that an arbitrary graph with edges labelled by the elements of $I$ is the graph of a chamber system if and only if $i$-adjacency is an equivalence relation for each $i \in I$. Thus, a $J$-residue of a chamber system over $I$ is itself the graph of a chamber system over $I-J$. By the usual abuse of notation, we shall often refer to a chamber system $(C, P)$ over $I$ by the mere symbol $C$. Also, in view of the fact that the chamber system can easily be recovered from its graph, we shall refer to the graph as the chamber system itself.

The motivating example is that of a chamber system $C(\Gamma)$ obtained from a geometry $\Gamma$ over $I$ in the following fashion. Take for the set of chambers the flags of $\Gamma$ of type $I$, and let two chambers $c, d$ be $i$-adjacent whenever $c \cap d$ is a flag of $\Gamma$ of type $I-\{i\}$. Conversely, given a chamber system $C$, we can define a geometry $\Gamma(C)$ by letting the objects of type $i$ of the geometry be the $(I-\{i\})$ components of $C$ and letting two objects be incident whenever they have a nonempty intersection.

In general, the two constructions need not be each other's inverse. However, if $\Gamma$ is a residually connected geometry over $I$, then there is a natural isomorphism from $\Gamma$ onto $\Gamma(C(\Gamma))$.

It is straightforward to see that a chamber system $C$ over $I$ is of the form $C(\Gamma)$ for some residually connected geometry $\Gamma$ over $I$ if and only if the following property holds for $C$ :

(*) For every subset $J$ of $I$ and every collection $\left(A_{i}\right)_{i \in J}$ of $(I-\{i\})$ components $A_{i}(i \in J)$ with $A_{i} \cap A_{j} \neq \varnothing$ for each $i, j \in J$, the intersection $\bigcap_{i \in J} A_{i}$ is a nonempty $(I-J)$-component.

A chamber system satisfying (*) is called residually connected. Taking $J=I$ in (*), we get $\{c\}=\bigcap_{i \in I} A_{i}$ for each chamber $c$, where $A_{i}$ is the $(I-\{i\})$ component containing $c$. Taking $J=\varnothing$, we get that such a chamber system is nonempty and connected.

A morphism of chamber systems is a map $\alpha: C \rightarrow C^{\prime}$, where $C$ and $C^{\prime}$ are chamber systems over the same index set $I$ and $\alpha$ preserves $i$-adjacency for all $i \in I$. In case $C$ and $C^{\prime}$ are residually connected, $\alpha$ corresponds to a morphism of the corresponding geometries.

Next, we introduce Coxeter diagrams for chamber systems. Following Tits 
[20], we call a chamber system $C$ over the set $\{i, j\}$ of size 2 a chamber system of type $m$ (for $m \in \mathbb{N}, m \geqslant 2$ ) if it is of the form $C(\Gamma$ ) where $\Gamma$ is a generalized $m$-gon. Let $M$ be a Coxeter matrix over $I$. For $n \geqslant 2$, a chamber system $C$ is said to be of type $M$ if it is connected and if for every subset $\{i, j\}$ of $I$ of size 2 each $\{i, j\}$ component is a chamber system of type $M(i, j)$.

Notice that a chamber system $C$ of type $m$ satisfies $C \cong C(\Gamma(C))$ and that $\Gamma(C)$ is a generalized $m$-gon. If $n \geqslant 3$ and $M$ is a Coxeter matrix over $l$, then the geometry $\Gamma(C)$ associated with a chamber system of type $M$ need not be a geometry of type $M$. The converse, however, does hold: if $\Gamma$ is a geometry of type $M$, then $C(\Gamma)$ is a chamber system of type $M$. It is readily verified that our notion of chamber system of type $M$ coincides with the one given by Tits [20].

\section{Sheaves}

In this section, $M$ is Coxeter matrix over $I$, and $J$ is a subset of $I$ with $I \backslash J$ of size $\geqslant 2$, and $\Gamma=(V, *, t)$ is a geometry over $I-J$. Given a set $\mathbf{F}$ of flags of $\Gamma$, we define an $I$-sheaf $\Sigma$ on $\Gamma$ over $\mathrm{F}$ to be a family of geometries $\Sigma(F)$ over $I-t(F)$ indexed by $F \in \mathbf{F}$ such that ${ }_{J} \Sigma(F)=\Gamma_{F}$ and such that for all $F \in \mathbf{F}$ and all flags $F^{\prime}$ of $\Gamma_{F}$ with $F \cup F^{\prime} \in \mathrm{F}$, we have

$$
\Sigma(F)_{F^{\prime}}=\Sigma\left(F \cup F^{\prime}\right) \text {. }
$$

(The idea of (pre-) sheaves on geometries stems from Aschbacher [1]; the present version however differs slightly from his and serves a different purpose.) An $I$-sheaf $\Sigma$ on $\Gamma$ over $\mathbf{F}$ is said to be a sheaf of type $M$ if $\Sigma(F)$ is of type $M \mid(I-t(F))$ for every $F \in \mathbf{F}$. Thus, $\Gamma$ is of $J$-truncated type $M$ if and only if there is a sheaf on $\Gamma$ of type $M$ over the set of all flags of corank 2 . Clearly, if $\Sigma$ is a sheaf on $\Gamma$ of type $M$ over a set of flags containing the empty flag, then $\Gamma$ is the $J$-truncation of the geometry $\Sigma(\varnothing)$ of type $M$. We wish to define sheaves of type $M$ on $\Gamma$ over flag sets that are as big as possible. The following construction shows how sheaves can be used to obtain interesting chamber systems. Suppose that $\mathbf{F}$ contains all flags of $\Gamma$ of corank at most 1 . Then given a sheaf $\Sigma$ on $\Gamma$ over $\mathbf{F}$ of type $M$, we construct a chamber system $C=C(\Sigma)$ in the following way:

The chambers of $C$ are the sets $F \cup S$ for $F \in C(\Gamma)$ and $S \in C(\Sigma(F))$. Let $c_{1}=$ $F_{1} \cup S_{1}$ and $c_{2}=F_{2} \cup S_{2}$ be two chambers with $F_{r} \in C(\Gamma)$ and $S_{r} \in C\left(\Sigma\left(F_{r}\right)\right)$ for $r=1$, 2. If $i \in J$, then $c_{1}$ and $c_{2}$ are $i$-adjacent if and only if $F_{1}=F_{2}$ and $S_{1}, S_{2}$ are $i$-adjacent in $C\left(\Sigma\left(F_{1}\right)\right)$. If $i \in I-J$, then $c_{1}$ and $c_{2}$ are $i$-adjacent if and only if $F_{1}$, $F_{2}$ are $i$-adjacent in $C(\Gamma)$ and $S_{1}=S_{2}$. (Note that the latter equation makes sense as both sides are flags of $\Sigma\left(F_{1} \cap F_{2}\right)$, while $F_{1} \cap F_{2} \in \mathbf{F}$.) It is immediate that the labelled graph thus defined is the graph of a chamber system $C$ over $I$. 
LEMMA 1. Let $K$ be a subset of $I-J$ of size 3. Suppose that $\Gamma$ is a geometry of $J$-truncated type $M$, and that the flag set $\mathbf{F}$ contains all flags whose type meets $K$ nontrivially. If $\Sigma$ is a sheaf on $\Gamma$ over $\mathrm{F}$ of type $M$, then $C=C(\Sigma)$ is a chamber system of type $M$ with the following three properties:

(i) Let ${ }_{J} C$ be the labelled graph (labels from $I-J$ ) whose vertices are the $J$ components of $C$ and in which two J-components are i-adjacent (for $i \in I$ $-J)$ whenever they are contained in the same $(J \cup\{i\})$-component of $C$. Then ${ }_{J} C \cong C(\Gamma)$. In particular, ${ }_{J} C$ is a residually connected chamber system with $\Gamma\left({ }_{J} C\right) \cong \Gamma$ and the following isomorphism of geometries holds: $\Gamma \cong{ }_{\mathrm{J}} \Gamma(C)$

(ii) For each subset $H$ of $I-J$ meeting $K$ nontrivially and each $H$-residue $A$ of $C$, there is a unique flag $F$ in $\mathbf{F}$ of type $H$ belonging to each chamber of $A$.

(iii) For each flag $F$ in $F$, there is a unique $t(F)$-residue $A$ of $C$ whose chambers contain $F$; it satisfies $A \cong C(\Sigma(F))$ and $\Gamma(A) \cong \Sigma(F)$.

Proof. Observe that $\mathbf{F}$ contains all flags of corank $\leqslant 1$ so that $C=C(\Sigma)$ is well defined.

(i) If $c_{1}=F_{1} \cup S_{1}$ and $c_{2}=F \cup S_{2}$, with $F_{r} \in C(\Gamma), S_{r} \in C\left(\Sigma\left(F_{r}\right)\right)$ for $r=1,2$, are $J$-connected (i.e. are in the same $J$-component of $C$ ), then, clearly, $F_{1}=F_{2}$. Therefore, the map $\alpha$, $C \rightarrow C(\Gamma)$, assigning to a $J$-component of $C$ the chamber of $\Gamma$ contained in any chamber of $C$ in this $J$-component, is well defined. Now, $\alpha$ is injective, for if $c_{1}=F_{1} \cup S_{1}$ and $c_{2}=F_{2} \cup S_{2}$ with $F_{r} \in C(\Gamma), S_{r} \in C\left(\Sigma\left(F_{r}\right)\right)$ for $r=1,2$ satisfy $F_{1}=F_{2}$, then $S_{1}, S_{2}$ are chambers of the residually connected chamber system $C\left(\Sigma\left(F_{1}\right)\right)$ over $J$, hence connected in this chamber system. Consequently $c_{1}$ and $c_{2}$ are $J$-connected in $C$.

Also, $\alpha$ is surjective, for, suppose $F$ is a chamber of $C(\Gamma)$. Then $\Sigma(F)$ is a residually connected geometry, so has a chamber $S$. The $J$-component of $F \cup S$ in $C$ maps under $\alpha$ onto $F$. We write $c \sim d$ to denote that the chambers $c, d$ are $i$ adjacent. Furthermore, $\alpha$ is a morphism of labelled graphs: Suppose $A, B$ are two $i$-adjacent $J$-components, where $i \in I-J$. Then there is a chain

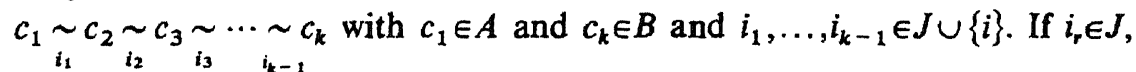
the $J$-components containing $c_{r}$ and $c_{r}$ and $c_{r+1}$ map onto the same element of $C(\Gamma)$ under $\alpha$. Since $C(\Gamma)$ is a chamber system, $i$-adjacency is an equivalence relation, so in order to derive $\alpha(A) \underset{i}{\sim} \alpha(B)$ it suffices to prove that $i_{r}=i$ (for $1 \leqslant r \leqslant k-1)$ implies that the chambers $F_{r}$ and $F_{r+1}$ of type $I-J$ contained in $c_{r}$ and $c_{r+1}$ respectively, are $i$-adjacent. But this is immediate from the definition of $c_{r} \sim c_{r+1}$. Finally we verify that $\alpha^{-1}$ is a morphism of labelled graphs. Suppose $F_{1} \sim F_{2}$ for two chambers $F_{1}, F_{2}$ of $C(\Gamma)$. Then $F_{1} \cap F_{2} \in \mathbf{F}$. 
By assumption $\Sigma\left(F_{1} \cap F_{2}\right)$ is residually connected, so if $\left\{x_{r}\right\}=F_{r}-\left(F_{1} \cap F_{2}\right)$ and $S_{r}$ is a chamber of $\Sigma\left(F_{r}\right)$ for $r=1,2$, the chambers $\left\{x_{r}\right\} \cup S_{r}(r=1,2)$ are in the same $(J \cup\{i\})$-component of $C\left(\Sigma\left(F_{1} \cap F_{2}\right)\right)$. This implies that the chambers $F_{r} \cup S_{r}=\left(F_{1} \cap F_{2}\right) \cup\left\{x_{2}\right\} \cup S_{r}(r=1,2)$ are in the same $(J \cup\{i\})$-component of $C$. Consequently, the $J$-components of $F_{r} \cup S_{r}$ are in the same $(J \cup\{i\})$ component of $C$. Since these $J$-components are $\alpha^{-1}\left(F_{r}\right)$, we are done. We have established that ${ }_{J} C$ and $C(\Gamma)$ are isomorphic labelled graphs. Since the latter is residually connected, ${ }_{J} C$ is a chamber system over $I-J$ with $\Gamma\left({ }_{J} C\right) \cong \Gamma(C(\Gamma)) \cong \Gamma$. Now, trivially, $\Gamma\left({ }_{J} C\right) \cong{ }_{J} \Gamma(C)$, so $\Gamma \cong \Gamma\left({ }_{J} C\right) \cong{ }_{J} \Gamma(C)$, whence (i).

(ii) Take $c \in A$. Then $c=F \cup S$ with $F \in C(\Gamma)$ and $S \in C(\Sigma(F))$. Let $F_{1}$ be the subflag of $F$ of type $H$. Suppose $d \in A$. Then $d=G \cup T$ with $G \in C(\Gamma)$ and $T \in C(\Sigma(G))$. Since $c, d$ are in the same $(I-H)$-component of $C$, they are $(I-H)$ connected. We want to show that $d$ contains $F_{1}$. By induction with respect to the length of an $(I-H)$ path from $c$ to $d$, it suffices to treat the case where $c \sim d$ for some $i \in I-H$. If $i \in J$, then $F_{1} \subseteq F=G \subseteq d$ and we are done. If $i \in I-$ $(H \cup J)$, then $F_{1} \subseteq F \cap G \subseteq G \subseteq d$ and we are done again. This proves (ii).

(iii) Let $F \in \mathbf{F}$. Set $H=t(F)$. Since $\Gamma$ is residually connected, there is a chamber $F_{1} \in C(\Gamma)$ with $F \subseteq F_{1}$, and, since $F_{1} \in \mathbf{F}$ and $\Sigma\left(F_{1}\right)$ is residually connected, there is a chamber $S_{1} \in C\left(\Sigma\left(F_{1}\right)\right)$ such that $c=F_{1} \cup S_{1}$ is a chamber in $C$ containing $F$. Define $A$ to be the $(I-H)$-component containing $c$. Let $d$ be a chamber in $C$ containing $F$. Then $d=G \cup T$ for some $G \in C(\Gamma), G \supseteq F$, and $T \in C(\Sigma(G))$. Since $\Gamma$ is residually connected, $F_{1}$ and $G$ are in the same $(I-(H \cup J)$-component of $C(\Gamma)$. By (i), this implies that $c$ and $d$ are in the same $((I-(H \cup J)) \cup J)$ component of $C$. Since $(I-(H \cup J)) \cup J=I-H$, this means that $d$ belongs to $A$. This proves the first statement of (iii).

Define a map $\beta: A \rightarrow C(\Sigma(F))$ as follows: $\beta(a)=R \cup S$ if $a=(F \cup R) \cup S$, where $R$ is a chamber of $\Gamma_{F}$ and $S$ is a chamber of $\Sigma(F \cup R)=\Sigma(F)_{R}$. Clearly, $\beta$ is injective. By the first statement of (iii), $\beta$ is surjective. Also, from the definition of $C$ it follows immediately that $\beta$ is a morphism. We next verify that $\beta^{-1}$ is a morphism. Suppose to this end that $R, R_{1}$ are chambers of $\Gamma_{F}$ and that $S \in C(\Sigma(F \cup R)), S_{1} \in C\left(\Sigma\left(F \cup R_{1}\right)\right)$, such that $R \cup S$ and $R_{1} \cup S_{1}$ are $i$-adjacent in $C(\Sigma(F))$ for some $i \in I-H$. If $i \in J$, then $R=R_{1}$, and $S, S_{1}$ are $i$-adjacent in $C(\Sigma(F \cup R))$, so again $\beta^{-1}(R \cup S)=F \cup R \cup S$ and $\beta^{-1}\left(R_{1} \cup S_{1}\right)=F \cup R \cup S_{1}$, are $i$-adjacent in $C$. If $i \in I-(H \cup J)$, then $S=S_{1}$ in $C\left(\Sigma\left(F \cup\left(R \cap R_{1}\right)\right)\right)$. The conclusion is that $\beta$ is an isomorphism of chamber systems over $I-H$, establishing $A \cong C(\Sigma(F))$. Finally, since $\Sigma(F)$ is residually connected, we have $\Gamma(C(\Sigma(F))) \cong \Sigma(F)$, whence $\Gamma(A) \cong \Sigma(F)$.

It remains to show that $C$ is a chamber system of type $M$. Since, $C \cong C(\Gamma)$ is connected, $C$ is connected. Let $\{i, j\}$ be a subset of $I$ of size 2 and let $A$ be an $\{i, j\}$-component of $C$. Set $H=I-(\{i, j\} \cup J)$. Then $H \cap K \neq \varnothing$ since $|K|=3$ 
and $H \subseteq I-J$, so by (ii), the $(\{i, j\} \cup J)$-component $A_{1}$ containing $A$ affords a unique flag $F$ of type $H$ belonging to each chamber of $A_{1}$. Now $F \in \mathbf{F}$, so by (iii) the $H$-residue $A_{1}$ satisfies $A_{1} \cong C(\Sigma(F))$. The $\{i, j\}$-component $A$ of $C$ is clearly an $\{i, j\}$-component of $A_{1}$. Since $A_{1} \cong C(\Sigma(F))$, this component is isomorphic to an $\{i, j\}$-component of $C(\Sigma(F))$. But $\Sigma(F)$ is of type $M_{1}$ so each $\{i, j\}$-component of $C(\Sigma(F))$, and hence $A$, is isomorphic to a chamber system of type $M(i, j)$. This ends the proof of the lemma.

LEMMA 2. Let $K$ be a subset of $I-J$ of size $\geqslant 3$ such that $\left.M\right|_{K}$ is neither $C_{3}$ nor $\mathrm{H}_{3}$. Suppose that $\Gamma$ is a geometry of $J$-truncated type $M$, and that $\mathbf{F}$ is a set of flags of $\Gamma$ containing each flag whose type meets $K$ nontrivially. If $\Sigma$ is a sheaf on $\Gamma$ over $\mathbf{F}$ of type $M$ such that for each $F \in \mathbf{F}$ each residue of type $C_{3}$ or $H_{3}$ of the geometry $\Sigma(F)$ is covered by a building, then $\Gamma$ is the $J$-truncation of the quotient geometry $\Delta / A$, where $\Delta$ is a building of type $M$ and $A$ is a group of automorphisms of $\Delta$ acting freely on the set of all flags of corank 2 and satisfying $(\mathrm{Q} 1)_{\mathrm{I}-\mathrm{K}}$ (cf. Theorem 2).

Proof. According to Lemma 1, there is a chamber system $C=C(\Sigma)$ of type $M$ with the properties (i), (ii), (iii) stated in that lemma.

According to Tits [20, Cor. 3], the universal 2-cover of $C$ is the chamber system of a building if and only if the universal 2 -cover of each $K$-component for $K$ a subset of $I$ of size 3 is the chamber system of a building of type $\left.M\right|_{K}$. By Tits [20, Cor. 4 and Prop. 6], our assumptions on $K, F$ and on residues of type $\mathrm{C}_{3}$ or $\mathrm{H}_{3}$, and Lemma 1 , this condition is satisfied. Thus, there exists a building $\Delta$ of type $M$ and a morphism $\alpha: C(\Delta) \rightarrow C$ of chamber systems which is the universal 2-cover of $C$.

Write $D=C(\Delta)$, and let $A$ be the group of deck transformations of the cover. Then $\alpha$ is the composition of the quotient map determined by $A$ and an isomorphism from $D / A$ to $C$. Without harming generality, we identify $D / A$ and $C$ by means of the latter isomorphism. The morphism $\alpha: D \rightarrow D / A=C$ can be used to define a morphism $\pi: \Delta \rightarrow \Gamma(C)$ as follows:

If $x$ is an object of type $i$, then it determines a unique $(I-\{i\})$-component $x_{*}$ of $D$, and this component is mapped by $\alpha$ onto the $(I-\{i\})$-component $\pi(x)=\alpha\left(x_{*}\right)$ of $C$. Since the action of $A$ on $D$ naturally translates into an action of $A$ on $\Delta$, we can also view $\pi$ as the quotient map $\pi: \Delta \rightarrow \Delta / A$. In particular, $\Gamma(C)=\Delta / A$. Since, according to Lemma 1 , the geometries $\Gamma$ and,$\Gamma(C)$ are isomorphic, we get $\Gamma \cong r(\Delta / A)$, as wanted.

By definition of 2-cover, if $a \in A$ fixes a flag of corank 2 of $\Delta$, then $a$ fixes all objects incident to it, and by connectivity of $D$, it follows that $a$ is the identity. This proves that $A$ acts freely on the set of all flags of corank 2 .

We finish by checking that the restriction $\pi_{F}$ of $\pi$ to the residue $\Delta_{F}$ induces an 
isomorphism

$$
\pi_{F}: \Delta_{F} / A_{F} \rightarrow(\Delta / A)_{\pi(F)},
$$

for each flag $F$ of $\Gamma$ whose type is not properly contained in $I-K$. It is obvious that $\pi_{F}$ is a surjective morphism.

In order to show that $\pi_{F}$ is injective we have to verify that if $x, y$ are two objects of $\Delta_{F}$ with $\pi(x)=\pi(y)$, then $x$ and $y$ have the same image in $\Delta_{F} / A_{F}$. Thus, let $x, y$ be objects of $\Delta_{F}$ of type, say $i$, with $\pi(x)=\pi(y)$. Let $x_{*}, y_{*}, F_{*}$ be the components of $D$ corresponding to $x, y, F$, respectively. Since $x, y$ are objects of $\Delta_{F}$, there exist chambers $c, d$ in $x_{*} \cap F_{*}, y_{*} \cap F_{*}$, respectively.

If we can choose $c, d$ such that $\alpha(c)=\alpha(d)$, then let $a \in A$ be a deck transformation sending $c$ to $d$. Since $F_{*}^{a}$ is an $(I-t(F))$-component of $D$ meeting $F_{*}$, we have $F_{*}^{a}=F_{*}$, so that $a \in A_{F}$ and $x^{a}=y$ as wanted.

If $\alpha(c) \neq \alpha(d)$ and $\alpha(c)=e_{0}, e_{1}, \ldots, e_{r}=\alpha(d)$ is a path in $C$ joining $\alpha(c)$ and $\alpha(d)$ entirely contained in a $(t(F) \cup\{i\})$-residue of $C$, then lifting this path to a path in $D$ starting in $c$, we find a chamber $c^{\prime} \in x_{*} \cap F_{*}$ with $\alpha\left(c^{\prime}\right)=\alpha(d)$, and we are done again. It remains to show that we can find such a path, i.e. that $\alpha(c)$ and $\alpha(d)$ belong to the same $(t(F) \cup\{i\})$-residue of $C$. Set $H=(t(F) \cup\{i\}) \cap K$. Observe that by the assumption on $\mathbf{F}$, the set $H$ is nonempty and that by the assumption on $x, y$ the chambers $\alpha(c)$ and $\alpha(d)$ contain the same flag, say $G$, of type $H$.

Now $G \in \mathbf{F}$, so in view of (ii), (iii) of Lemma 1, the chambers $\alpha(c), \alpha(d)$ are contained in the same $H$-residue $A$ of $C$, while $A \cong C(\Sigma(G))$. But $\Sigma(G)$ is residually connected and $\alpha(c), \alpha(d)$ are contained in the same object of $\Gamma(A) \cong \Sigma(G)$ of type $j$ for each $j \in t(F) \cup\{i\}$ whence $\alpha(c)$ and $\alpha(d)$ are contained in the same $(t(F) \cup\{i\})$-residue. This shows that $\pi_{F}$ is injective.

Finally, we have to show that $\pi_{F}$ preserves nonincidence. Let $x, y$ be objects of $\Delta_{F}$. If $\pi(x)$ and $\pi(y)$ are incident objects of $(\Delta / A)_{\pi(F)}$, there is a chamber contained in $\pi(x), \pi(y), \pi(F)$. Hence there exists a chamber in $D$ contained in $x_{*}$, $y_{*}^{\prime}, F_{*}$ for some object $y^{\prime}$ of $\Delta$ with $\pi\left(y^{\prime}\right)=\pi(y)$. By injectivity of $\pi_{F}$, the objects $y, y^{\prime}$ have the same image in $\Delta_{F} / A_{F}$, so that the image of $x$ and $y$ in $\Delta_{F} / A_{F}$ are incident.

We conclude that $\pi_{F}$ is an isomorphism. This ends the proof of Lemma 2.

\section{PROOF OF THEOREM 2}

Let $I, J$, and $M$ be as in the statement of the theorem, and suppose that $\Gamma=$ $(V, *, t)$ is a geometry of $J$-truncated type $M$. For $i \in I-J$, denote by $\mathbf{F}^{i}$ the set of all flags whose type contains $i$. Furthermore, let $\mathbf{F}$ be the set of all nonempty flags on $\Gamma$. In view of Lemma 2, it suffices to construct a sheaf $\Sigma$ on $\Gamma$ over $F$ of type $M$. 
Let $x$ be an object of $\Gamma$ of type 0 . By a remark made above, the residue $\Gamma_{x}$ is a geometry of $J$-truncated type $A_{n-1}$ (up to a labelling of the nodes, that is), so that by Theorem 1, there exists a unique geometry $\Sigma(x)$ of type $\left.M\right|_{(I-\{0\})}$ with $\Gamma_{x}={ }_{J} \Sigma(x)$. For the sake of future identification, we shall view $\Sigma(x)$ as the geometry whose objects of type $i$ for $i>3$ are collections of objects of type 3 (i.e. of planes in $\Sigma(X)$ ), incidence between objects of type $>3$ being symmetrized containment and incidence between an object $y$ of $\Gamma_{x}$ and an object $S$ of $\Sigma(x)$ of type $>3$ being the existence of $w \in S$ with $w * y$. It is well known and not hard to prove that this is indeed a faithful representation of $\Sigma(x)$.

Consider an object $y$ of $\Gamma$ of type 1. If $M=D_{n}$, then define $\Sigma(y)$ in the same manner as $\Sigma(x)$ above. If $M=C_{n}$, then set

$$
\Sigma(y)={ }^{\{0\}} \Gamma_{y} \oplus \Sigma(x)_{y},
$$

where $x$ is an object of ${ }^{\{0\}} \Gamma_{y}$ and $\oplus$ stands for the direct sum of geometries. Notice that this definition does not depend on the choice of $x$, since $\Sigma(x)$, is a geometry of type $A_{n-2}$ (up to relabelling of types) and is therefore uniquely determined by its $J$-truncation $J(x)_{y}=\Gamma_{\{x, y\}}={ }^{\{2,3\}} \Gamma_{y}$. Also notice that

$$
\Gamma_{y}={ }^{00\}} \Gamma_{y} \oplus \Gamma_{\{x, y\}}={ }_{j} \Sigma(y) \text {. }
$$

From now on, we again allow for $M=C_{n}$ or $D_{n}$. Given a flag $\{x, y\}$ with objects $x, y$ of type 0,1 , respectively, we have

$$
\Sigma(x)_{y}=\Sigma(y)_{x} .
$$

For, both sides of the equation pertain to geometries of type $A_{n-2}$ (up to relabelling of types), whence they are both uniquely determined by their $J$ truncations, while

$$
{ }_{J} \Sigma(x)_{y}=\Gamma_{\{x, y\}}={ }_{J} \Sigma(y)_{x} .
$$

As a consequence, $\Sigma(F)$ for $F \in \mathbf{F}^{0} \cup \mathbf{F}^{1}$ can be (well) defined by $\Sigma(F)=$ $\Sigma(x)_{\{F-\{x\}\}}$ if $x \in F$ has type 0 or 1 . We proceed with an object $z$ of type 2. Set

$$
\Sigma(z)=\{0,1\} \Gamma_{z} \oplus \Sigma(F)_{z},
$$

where $F$ is a flag of $\Gamma_{z}$ of type $\{0,1\}$. In order to show that this is well defined, i.e. independent of the choice of $F$, let $F^{\prime}$ be another flag of $\Gamma_{2}$ of type $\{0,1\}$. If $F$ and $F^{\prime}$ are $i$-adjacent in $C\left(^{(0,1\}} \Gamma_{z}\right)$ for some $i \in\{0,1\}$, then

$$
\Sigma(F)_{z}=\Sigma\left(F \cap F^{\prime}\right)_{(F-F) \cup\{z\}}=\Sigma\left(F \cap F^{\prime}\right)_{\{F-F) \cup\{z\}}=\Sigma\left(F^{\prime}\right)_{z},
$$

so that $\Sigma(F)_{z}=\Sigma\left(F^{\prime}\right)_{z}$.

If $F$ and $F^{\prime}$ are not $i$-adjacent in $C\left({ }^{(0.1)} \Gamma_{z}\right)$, in view of connectedness of the latter chamber system (due to residual connectedness of $\Gamma$ ), we can find a path 
from $F$ to $F^{\prime}$ in $C\left({ }^{(0,1\}} \Gamma_{z}\right)$ and obtain $\Sigma(F)_{z}=\Sigma\left(F^{\prime}\right)_{z}$ again by repeated application of the above argument along the edges of the path. The conclusion is that $\Sigma(z)$ is well defined indeed. Obviously, $\Gamma_{z}=\{0,1\} \Gamma_{z} \oplus_{J} \Sigma(F)_{z}=, \Sigma(z)$. Now let $x, y, z$ be mutually incident objects of type $0,1,2$, respectively. Then

$$
\Sigma(x)_{z}=\Sigma(z)_{x} \quad \text { and } \quad \Sigma(y)_{z}=\Sigma(z)_{y}
$$

For,

$$
\Sigma(z)_{x}=\{1\} \Gamma_{\{x, z\}} \oplus \Sigma(\{x, y\})_{z}=\left({ }^{\{1} \Sigma(x)_{z} \oplus \Sigma(y)_{\{x, z\}}=\Sigma(y)_{z}\right.
$$

and

$$
\Sigma(z)_{y}={ }^{\{0\}} \Gamma_{\{y, z\}} \oplus \Sigma(\{x, y\})_{z}={ }^{\{0\}} \Gamma_{y} \oplus \Sigma(y)_{\{x, z\}}=\Sigma(y)_{z} .
$$

Again, we can define $\Sigma(F)=\Sigma(x)_{F-\{x\}}$ for $F \in F^{0} \cup F^{1} \cup F^{2}$, where $x \in F$ has type $t(x)<3$. Finally, for $w$ an object of $\Gamma$ of type 3, set $\Sigma(w)=\Gamma_{w} \oplus \Sigma(F)_{w}$, where $F$ is a flag of type $\{0,1,2\}$ in $\Gamma_{w}$. By arguments completely analogous to those for $z$ above, we get $\Gamma_{w}={ }_{J} \Sigma(w)$ and $\Sigma(w)_{u}=\Sigma(u)_{w}$ for all $u \in \Gamma_{w}$. By now, it is obvious that $\Sigma(u)$ is a geometry of type $\left.M\right|_{(I-\{i\})}$ if $u$ is an object of type $i \in\{0,1,2,3\}$. It readily follows that $\Sigma$ is a sheaf of type $M$ over $F=$ $F^{0} \cup F^{1} \cup F^{2} \cup F^{3}$. This settles the theorem.

\section{Proof of COROLlary 1}

Retain the notation of the above proof. Since all residues of ${ }_{J} \Delta$ of rank 2 are isomorphic to corresponding residues of $\Gamma$, they are thick and finite. Therefore, $\Delta$ is a thick finite building of type $M$. In the remainder of this proof, the objects of type 0 are called points and the objects of type 1 if $M=C_{n}$, and of type 2 if $M=D_{n}$, are called lines. By Lemma 5 of Brouwer and Cohen [2] and a straightforward verification of the hypotheses of that lemma applied to the collinearity graph of ${ }^{H} \Delta$ where $H=\{0,1\}$ if $M=C_{n}$ and $H=\{0,2\}$ if $M=D_{n}$ (in fact, this graph is a dual polar graph if $M=C_{n}$ and a halved dual polar graph if $M=D_{n}$; see also Brouwer and Cohen [3]) it follows that for each $a \in A$ there is a point $x$ of $\Delta$ such that $x$ is collinear with $x^{a}$. If $x=x^{a}$, then $a$ restricted to $\Delta_{x}$ is an automorphism of a projective space. But the restriction $\pi_{\{x\}}$ must be an isomorphism since the corresponding map $C\left(\Delta_{x}\right) \rightarrow C(\Sigma(x))$ is a 2-cover of chamber systems and both $\Delta_{x}$ and $\Sigma(x)$ are buildings. Consequently, $a$ induces the identity on $\Delta_{x}$. Similarly, if $a$ fixes a line $l$, it induces the identity on $\Delta_{l}$. By residual connectedness of ${ }^{\{0,1\}} \Delta$, it follows that the identity is the only transformation in $A$ fixing either a point or a line.

Again, given $a \in A$, consider a point $x$ of $\Delta$ such that $x$ and $x^{a}$ are both incident to a line, $l$ say. Then by $(\mathrm{Q} 1)_{J}$ applied to the flag $F=\{l\}$, there is an 
element $b \in A_{F}$ such that $x^{a}=x^{b}$. But by the above, $b$ must be the identity, so that $x^{a}=x$ and, again by the above, $a$ is the identity. The conclusion is that $A$ is trivial, whence $\Gamma \cong{ } \Delta$, so that $\Gamma$ is the $J$-truncation of a building of type $M$. This proves the corollary.

\section{TWO APPLICATIONS; PROOF OF THE MAIN THEOREM}

A near polygon is a connected partial linear space $\Gamma=(X, L)$ such that for any point $x \in X$ and line $l \in L$, there is a unique point on $l$ nearest $x$. (Here, distances are measured in the collinearity graph.) For details on near polygons, the reader is referred to Brouwer and Wilbrink [4] and Shult and Yanushka [19]. A subset $Y$ of $X$ is called a subspace if for any two distinct collinear points in $Y$ every point on the line joining them is in $Y$. The subset $Y$ of $X$ is called geodesically closed if for any two points in $Y$ the points of every shortest path from one to the other are contained in Y. A quad is a geodesically closed subspace of diameter 2 which is nondegenerate (i.e. there is no point collinear with every point). It follows that a quad, with the collection of lines that it contains, has the structure of a generalized quadrangle. A hex is a geodesically closed subspace of diameter 3. We say that a near polygon is thick if each line has at least three points and if any two points at mutual distance 2 have at least three common neighbours. According to Shult and Yanushka [19], a thick near polygon has quads, i.e. each pair of concurrent lines is contained in a unique quad. Brouwer and Wilbrink [4] have shown that in a thick near polygon each pair of points at mutual distance $j$ is contained in a unique geodesically closed subspace of diameter $j$ (see also Shult $[18, \S 7]$ ). A near polygon is called nearly classical if it is thick and if for each point $x \in X$ and each hex $H$ containing $x$, the linear space of the lines and quads on $x$ contained in $H$ is a projective plane. (This concept is taken from Shad and Shult [17], see Shult [18].) Typical examples of near polygons of diameter $n$ are dual polar spaces, i.e. $\{2, \ldots, n-1\}$-truncations of buildings of type $C_{n}$, in which the points are the objects of type 0 and the lines are the objects of type 1. Cameron [9] has characterized these spaces. For $n=3$, his result states that nearly classical hexes are dual polar spaces. The local rank of $\Gamma$ at $x$ is the rank of $L_{x}$, i.e. the maximum size of a chain (totally ordered by inclusion) of proper nonempty subspaces of $L_{x}$.

PROPOSITION 1. Let $\Gamma$ be a nearly classical near polygon with finite local rank $n-1$ at some point and of diameter at least 3. Then the geometry over $\{0,1,2,3\}$ whose objects of type $0,1,2,3$ are the points, lines, quads and hexes of $\Gamma$, respectively, and in which incidence is given by symmetrized containment, is of $\{4, \ldots, n-1\}$-truncated type $C_{n}$. 
Proof. Let $x$ be a point where the local rank is $n-1$. Consider the local space $L_{x}$ of lines, quads and hexes on $x$. Since $\Gamma$ is nearly classical, every two quads on $x$ contained in a hex meet in a line. Consequently, $L_{x}$ is a truncation of a geometry of type $A_{n-1}$.

If $y$ is collinear to $x$, then the linear space $L_{l}$ of quads and hexes on the line $l$ containing both $x$ and $y$ is a projective space (in fact a residue in both $L_{x}$ and $L_{y}$ ) and $\operatorname{dim} L_{y}=1+\operatorname{dim} L_{l}=n-1$ so that by connectedness of $\Gamma$ the local rank is $n-1$ at each point. All remaining checks are straightforward.

COROLLARY 2. A nearly classical near polygon of finite local rank $n-1$ at some point, is isomorphic to the quotient $\Delta / A$ of $a$ dual polar space of rank $n$ by a group $A$ of automorphisms of $\Delta$ such that for each $a \in A, a \neq 1$, and each point $x$ the distance in the collinearity graph between $x$ and $x^{a}$ is at least 8. In particular, any finite nearly classical near polygon is a dual polar space.

Proof. By the above proposition and Theorem 1, there is a building of type $C_{n}$ admitting a group $A$ of automorphisms with the properties specified in Theorem 2. It is easy to see that the action of $A$ on the building induces an action of $A$ on its $\{2, \ldots, n-1\}$-truncation $\Delta$ as described. This leads to the first statement. The last statement follows from Corollary 1 .

It should be noted that in the hypotheses one does not need the full strength of the near polygon axiom. Let us call a connected partial linear space a 3-weak near polygon when for any two points $x, y$ at mutual distance at most 3 and any line $l$ on $y$ there is a unique point on $l$ nearest $x$. It is left for the reader to verify that for thick 3-weak near polygons, quads can be constructed as in [19]. The notion of nearly classical can be defined as in [17], and the hexes can be constructed as in [18] or [4]. The proof of Corollary 2 remains valid in this situation and establishes part (i) of the main theorem. Shult $[18,87]$ states that $A$ is trivial if one uses the full stength of the near polygon axiom. It would be of interest to have a direct proof of this fact using Corollary 2.

The second application that we shall deal with concerns the characterization of the halved dual polar spaces of rank $n$, i.e. the truncation of type $\{0,2\}$ of buildings of type $D_{n}$ in which the points and lines are the objects of type 0 and 2, respectively. For each field $K$ there is a unique thick building of type $D_{n}$ whose rank 2 residues are generalized digons or projective planes over $K$; we shall denote this building by $D_{n}(K)$. The truncation ${ }^{\{0,2\}} D_{n}(K)$ is a parapolar space as defined in Cohen and Cooperstein [11], when the objects of type 0 and 2 are viewed as points and lines respectively. This means that it is a connected partial linear space, all of whose lines have at least three points, such that

(i) if $l$ is a line and $x$ is a point collinear with two points on $l$, then $x$ is collinear with all points on $l$; 
(ii) for any two collinear points, the graph on their common neighbors is not a clique;

(iii) for any two points at mutual distance 2, the set of their common neighbors is either a singleton or carries the structure of a nondegenerate polar space of rank at least 2.

Moreover, it satisfies the axioms (P3) and (P4) (defined in [11]) for $k=3$. For convenience, we quote them here.

(P3) $)_{k} \quad$ For any two points at mutual distance 2 , the set of their common neighbors carries the structure of a nondegenerate polar space of rank $k$.

(P4) If two points $x, y$ are at mutual distance 2 and have at least two common neighbors and if $l$ is a line on $y$ with no points collinear to $x$, then the set of points collinear to $x$ and to all points ofl is either empty or a maximal clique in the collinearity graph on the set of common neighbors of $x$ and $y$.

Observe that maximal cliques are subspaces due to (i); they are called maximal singular subspaces. The singular rank of a parapolar space $\Gamma$ is the maximal rank of $S$ for $S$ ranging over all maximal singular subspaces of $\Gamma$.

PROPOSITION 2. Let $\Gamma$ be a parapolar space of finite singular rank $n \geqslant 4$. If $\Gamma$ satisfies $(\mathrm{P} 3)_{3}$ and $(\mathrm{P} 4)$, then the geometry over $\{0,1,2,3\}$ whose objects of type $0,1,2,3$ are the points, maximal singular subspaces of rank $n$, lines, maximal singular subspaces of rank 3 , respectively, and in which incidence is given by symmetrized containment, is of $\{4, \ldots, n\}$-truncated type $D_{n+1}$.

Proof. By Theorem 1 of Cohen and Cooperstein [11], it is immediate that the geometry over $\{0,1,2,3\}$ described in the statement of the proposition admits a sheaf on $\Gamma$ over the set $F$ of all nonempty flags, which is of type $D_{n+1}$. This implies the proposition.

The following corollary establishes part (ii) of the main theorem. We shall denote the parapolar space associated with ${ }^{\{0,2\}} D_{n}(K)$ as above, by $D_{n, 0}(K)$.

COROLLARY 3. Let $\Gamma$ be a parapolar space of finite singular rank $n-1 \geqslant 4$. If $\Gamma$ satisfies $(\mathrm{P} 3)_{3}$ and $(\mathrm{P} 4)$, then it is isomorphic to the quotient $D_{n, 0}(K) / A$, where $K$ is a field and $A$ is a group of automorphism of $D_{n}(K)$ such that for each $a \in A$, $a \neq 1$, the distance between $a$ point and its image under $a$ in the collinearity graph of $D_{n, 0}(K)$ is at least 5.

Proof. This can be obtained by combining the above proposition and Theorem 2.

As stated in the introduction, the corollary generalizes Cooperstein's main 
theorem in [12]. It can also be used to strengthen Theorem 1 in Cohen and Cooperstein [11]. We shall only bother to present a finite version of the latter theorem. For this purpose, we need some more notation.

Let $n=6,7,8$. Just as for type $D_{n}$, to each field $K$ corresponds a unique thick building of type $E_{n}$ whose rank 2 residues are generalized digons or projective planes over $K$; we shall denote this building by $E_{n}(K)$.

The spaces corresponding to the rank 2 geometries ${ }^{\{0,1\}} E_{6}(K),{ }^{\{5,6\}} E_{7}(K)$ with point set the set of objects of type 0,6 , respectively, will be denoted by $E_{6,0}(K), E_{7,6}(K)$, respectively. Finally, let $A_{n, d}(K)$, where $K$ is field, $n \geqslant 3$ and $1 \leqslant d \leqslant n-2$, denote the parapolar space associated with the projective space $A_{n}(K)$ of rank $n$ defined over $K$ whose points are the subspaces of rank $d$ and whose lines are the sets of subspaces of rank $d$ incident to 'a flag of type $\{d-1, d+1\}$, i.e. an incident pair consisting of a subspace of rank $d-1$ and a subspace of rank $d+1$.

THEOREM 3. Let $k \geqslant 2$ and let $\Gamma$ be a finite parapolar space which is not a polar space. Then $\Gamma$ satisfies $(\mathrm{P} 3)_{k}$ and $(\mathrm{P} 4)$ if and only if there exists a finite field $K$ such that $\Gamma$ is isomorphic to one of $A_{n, d}(K)($ for $1 \leqslant d \leqslant n-2), D_{n, 0}(K),($ for $n \geqslant 4), E_{6,0}(K), E_{7,6}(K)$.

Proof. Immediate from Theorem 1 in Cohen and Cooperstein [11] and Corollary 3.

\section{CONCLUding REMARK}

There are many other truncated Coxeter types for which analogues of Theorem 2 exist. We shall only mention the following generalization of the case $M=D_{n}$ of Theorem 2 . Let $M$ be the Coxeter diagram

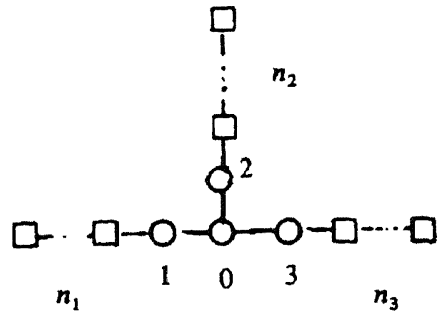

over $I=\{0,1, \ldots, n-1\}$, where $n=4+n_{1}+n_{2}+n_{3}$ and $n_{1}, n_{2}, n_{3} \geqslant 0$. Set $K=\{0,1,2,3\}$ and $J=I-K$.

THEOREM 4. Any geometry of $J$-truncated type $M$ is the $J$-truncation of the quotient geometry $\Delta / A$, where $\Delta$ is a building of type $M$ and $A$ is a group of 
automorphisms of $\Delta$ acting freely on the set of all flags of rank 2 and satisfying (Q1), (cf. Theorem 2).

Notice that the theorem includes truncations of $D_{n}, E_{6}, E_{7}, E_{8}$ as special cases. In particular, it can be shown that any thick finite geometry of truncated type

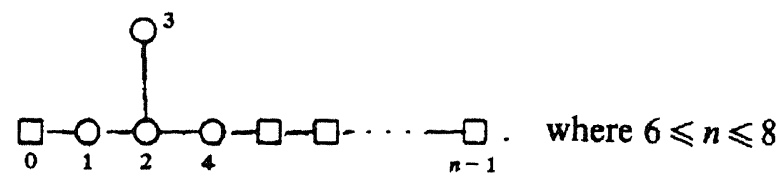

is a truncation of a thick finite building of type $E_{n}$. The proofs are omitted; they are similar to those of Theorem 2 and Corollary 1.

\section{REFERENCES}

1. Aschbacher, M., 'Flag Structure on Tits Geometries', Geom. Dedicata 14 (1983), 21-32.

2. Brouwer, A. E. and Cohen, A. M., 'Some Remarks on Tits Geometries', Indag. Math. 45 (1983), 393-402.

3. Brouwer, A. E. and Cohen, A. M., 'Computation of some Parameters of Lie Geometries' Annals of Discrete Math. 26 (1985), 1-48.

4. Brouwer, A. E. and Wilbrink, H. A., 'The Structure of Near Polygons with Quads', Geom. Dedicata 14 (1983), 145-176.

5. Buekenhout, F., 'A Characterization of Polar Spaces', Simon Stevin 53 (1979), 3-7.

6. Buekenhout, F., 'Diagrams for Geometries and Groups', J. Comb. Theory (A) 27 (1979), 121 151.

7. Buekenhout, F. and Hubaut, X., 'Locally Polar Spaces and Related Rank 3 Groups', J. Algebra 45 (1977), 391-434.

8. Buekenhout, F. and Shuit, E. E., 'On the Foundations of Polar Geometry', Geom. Dedicata 3 (1974), 155-170.

9. Cameron, P. J., 'Dual Polar Spaces', Geom. Dedicata 12 (1982), 75-85.

10. Cohen, A. M., 'An Axiom System for Metasymplectic Spaces', Geom. Dedicata 12 (1982), 417 433.

11. Cohen, A. M. and Cooperstein, B. N., 'A Characterization of some Geometries of Exceptional Lie Type', Geom. Dedicata 15 (1983), 73-105.

12. Cooperstein, B. N. 'A Characterization of a geometry related to $\Omega_{2 m}^{+}(K)^{\prime}$ (to appear in J. Comb. Theory).

13. Hall, J. I. and Shult, E. E., 'Locally Cotriangular Graphs (to appear)

14. Johnson, P. and Shult, E. E., 'Local Characterizations of Polar Spaces' (to appear in Geom. Dedicata)

15. Kantor, W. M., 'Locally Polar Lattices', J. Comb. Theory (A) 26 (1979), 90-95.

16. Ronan, M. A., 'Extending Locally Truncated Buildings and Chamber Systems' (preprint revised version).

17. Shad, S. and Shult, E. E., 'The Near n-gon Geometries' (preprint).

18. Shult, E. E., 'Characterizations of the Lie Incidence Geometries', in Surveys in Combinatorics, LMS Lecture Note Series 82 (ed. E. K. Lloyd), Cambridge Univ. Press, Cambridge, 1983, pp. 157-186.

19. Shult, E. E. and Yanushka, A., 'Near $n$-gons and Line Systems', Geom. Dedicata 9(1980), 1-72. 
20. Tits, J., 'A Local Approach to Buildings', in The Geometric Vein (the Coxeter Festschrift), Springer-Verlag, Berlin, 1981, pp. 519-547.

Authors' address:

CWI,

Kruislaan 413,

1098 SJ Amsterdam, The Netherlands

(Received, August 19, 1983; final version, January 23, 1985) 